Mathematical Modelling and Analysis

Volume 21 Number 2, March 2016, 143-158

http://dx.doi.org/10.3846/13926292.2016.1137503

(c) Vilnius Gediminas Technical University, 2016
Publisher: Taylor\&Francis and VGTU

http://www.tandfonline.com/TMMA

ISSN: $1392-6292$

eISSN: $1648-3510$

\title{
Effect of Time Delay on Spatial Patterns in a Airal Infection Model with Diffusion
}

\author{
Jia Liu ${ }^{a}$, Qunying Zhang ${ }^{b}$ and Canrong $\operatorname{Tian}^{c}$ \\ ${ }^{a}$ School of Mathematics and Physics, Changzhou University \\ 213164 Changzhou Jiangsu, China \\ ${ }^{b}$ School of Mathematical Science, Yangzhou University \\ 225002 Yangzhou, China \\ ${ }^{c}$ Department of Basic Sciences, Yancheng Institute of Technology \\ 224003 Yancheng, China \\ E-mail: tcr_2001@sohu.com
}

Received June 11, 2015; revised December 27, 2015; published online March 15, 2016

\begin{abstract}
This paper is concerned with the dynamics of a viral infection model with diffusion under the assumption that the immune response is retarded. A time delay is incorporated into the model described the delayed immune response after viral infection. Based upon a stability analysis, we demonstrate that the appearance, or the absence, of spatial patterns is determined by the delay under some conditions. Moreover, the spatial patterns occurs as a consequence of Hopf bifurcation. By applying the normal form and the center manifold theory, the direction as well as the stability of the Hopf bifurcation is explored. In addition, a series of numerical simulations are performed to illustrate our theoretical results.
\end{abstract}

Keywords: viral infection, diffusion, time delay, spatial patterns.

AMS Subject Classification: 35B36; 35K57; 92D30.

\section{Introduction}

Mathematical modelling of viral dynamics has attracted the interest of experimentalists and theorists alike during the last few decades [10,11, 13, 21, 28, 31]. This is because viral models can provide insights into the dynamics of viral load in vivo and may play a significant role in the development of a better understanding of diseases and various drug therapy strategies against them. During viral infections, it is well-known and pointed out by the work of $[5,17]$ that viral reproduction always involves host cells and uses the cellular machinery for the synthesis of their genome and other components. On the other hand, Bartholdy et al. [2] and Wodarz et al. [30] found that the turnover of free virus is much faster than that of infected cells, which allowed them to make a quasisteadystate assumption, that is, the amount of free virus is simply proportional 
to the number of infected cells. Hence, Bonhoeffer et al. presented the simplified model in [4]. Antibodies, cytokines, natural killer cells, and T cells are essential components of a normal immune response to a virus. In particular, cytotoxic T lymphocytes (CTLs) play a key role in antiviral defense by attacking virus-infected cell, and it is also believed that they are the main host immune factor that limits the extent of virus replication in vivo and thus determines virus load [34]. Therefore, the population dynamics of viral infection with CTL response has been paid much attention in the last few decades.

Most of them use ordinary differential equations to describe different aspects of the dynamics of the host-parasite interaction, and in some sense, delay models give better compatibility with reality, as they capture the dynamics from the time of infection to the infectiousness. For example, during HIV infection, the intracellular phase is about 0.9 days, but the average half-life of plasma virus is only around $6 \mathrm{~h}$ [19]. Thus, delays should be incorporated into the infection equation and/or the virus production equation of a model to account for effect of intracellular delay which leads to mathematical models by delay differential equation (DDE). Many authors have studied the mathematical modeling of viral dynamics with CTL immune response in the literature, which are given by systems of ordinary differential equation (ODE) and DDE $[8,14,20,22,29]$.

Based on the above discussion, Wang et al. [26] proposed a basic mathematical model:

$$
\left\{\begin{array}{l}
\frac{d u_{1}}{d t}=\lambda-d u_{1}-\beta u_{1} u_{2}, \\
\frac{d u_{2}}{d t}=\beta u_{1} u_{2}-a u_{2}-p u_{2} u_{3}, \\
\frac{d u_{3}}{d t}=c u_{2}(\tau)-b u_{3},
\end{array}\right.
$$

where $u_{1}, u_{2}, u_{3}$ represent the densities of susceptible host cells, free virus and cytotoxic T lymphocytes (CTLs). Susceptible host cells are generated at a rate $\lambda$, die at a rate $d u_{1}$ and become infected by the virus at a rate $\beta u_{1} u_{2}$. Infected cells die at a rate $a u_{2}$ and are killed by the CTL response at a rate $p u_{2} u_{3}$, corresponding to lytic effector mechanisms of CTL response. The CTL response is activated at a rate proportional to the number of infected cells at a previous time $c u_{2}(\tau)=c u_{2}(t-\tau), \tau$ is a positive constant accounting for the time delay of CTL response, and also decays exponentially at a rate proportional to its current strength $b u_{3}$. It is assumed that the parameters in system (1.1) are positive constants.

Note that model (1.1) implicitly assumes that cells and viruses are well mixed, and ignores the mobility of cells, viruses and CTLs, whereas biological motion plays a crucial role in many biological phenomena. In fact, many investigators have introduced population movements into related equations for ecological, epidemiological and tumorigenic modelling and simulations in efforts to understand the most basic features of spatially distributed interactions $[7,12,16,18,25,27]$. But so far, there have been few studies to consider the influences of spatial structures on virus dynamics. Thus, we introduce the 
random mobility for cells, viruses and CTLs into (1.1):

$$
\begin{cases}u_{1 t}-d_{1} \Delta u_{1}=\lambda-d u_{1}-\beta u_{1} u_{2}, & (\mathbf{x}, t) \in \Omega \times(0, T), \\ u_{2 t}-d_{2} \Delta u_{2}=\beta u_{1} u_{2}-a u_{2}-p u_{2} u_{3}, & (\mathbf{x}, t) \in \Omega \times(0, T), \\ u_{3 t}-d_{3} \Delta u_{3}=c u_{2}(\tau)-b u_{3}, & (\mathbf{x}, t) \in \Omega \times(0, T), \\ \partial u_{1} / \partial \eta=\partial u_{2} / \partial \eta=\partial u_{3} / \partial \eta=0, & (\mathbf{x}, t) \in \partial \Omega \times(0, T), \\ u_{i}(\mathbf{x}, t)=\varphi_{i}(\mathbf{x}, t)(i=1,2,3), & (\mathbf{x}, t) \in \Omega \times[-\tau, 0] .\end{cases}
$$

$\Omega$ is a bounded domain in $\mathbb{R}^{n}$ with smooth boundary $\partial \Omega, \eta$ is the outward unit normal vector on the boundary. The homogeneous Neumann boundary condition implies that the above system is self-contained and there is no infection across the boundary. The positive constants $d_{i}(i=1,2,3)$ are the free diffusion coefficients corresponding to $u_{i}(i=1,2,3)$. The initial function $\varphi_{i}(i=1,2,3)$ is positive, Hölder continuous and satisfies $\partial \varphi_{i} / \partial \eta=0$ on the boundary.

Denote $\mathbf{u}=\left(u_{1}, u_{2}, u_{3}\right)$ and

$$
G(\mathbf{u})=\left(\begin{array}{l}
G_{1}(\mathbf{u}) \\
G_{2}(\mathbf{u}) \\
G_{3}(\mathbf{u})
\end{array}\right)=\left(\begin{array}{l}
\lambda-d u_{1}-\beta u_{1} u_{2} \\
\beta u_{1} u_{2}-a u_{2}-p u_{2} u_{3} \\
c u_{2}(\tau)-b u_{3}
\end{array}\right)
$$

It is clear that problem (1.2) always has two equilibria. The first is $E_{0}=$ $(\lambda / d, 0,0)$ and it represents the state in which the virus is absent. Further, denote $R_{0}=\lambda \beta /(a d)$, here $R_{0}$ is called the basic reproduction ratio of system (1.2). It is easy to show that if $R_{0}>1$, system (1.2) admits a unique endemic equilibrium $\mathbf{u}^{*}=\left(u_{1}^{*}, u_{2}^{*}, u_{3}^{*}\right)$, which is described by the following expressions

$$
\begin{aligned}
& u_{1}^{*}=\frac{c \lambda}{c d+b \beta u_{3}^{*}}, \quad u_{2}^{*}=\frac{b u_{3}^{*}}{c}, \\
& u_{3}^{*}=\frac{-(p c d+a b \beta)+\sqrt{(p c d+a b \beta)^{2}-4 b c p \beta(a d-\lambda \beta)}}{2 b p \beta},
\end{aligned}
$$

this represents the state in which the virus is present. The objective of this paper is to study the stability of the endemic equilibrium $\mathbf{u}^{*}$ when $\tau>0$, thus, throughout this paper, we always assume that $R_{0}>1$ holds.

Spatial patterns have attracted great attention recently. Diffusion-driven spatial patterns, also called Turing patterns, have been extensively studied in the modelling of many physical, chemical and biological processes $[1,3,15,18$, $23,24,25,27]$. While, not only diffusion can generate spatial patterns, but also time delay can induce spatial patterns $[6,32,33,35]$. For example, Zhang et al. [35] investigated that the time delay can not only cause spatially homogeneous Hopf bifurcation but also give rise to spatially heterogeneous ones in a delayed predator-prey diffusion system. Yan [32] demonstrated the stability of the positive equilibrium and the existence of Hopf bifurcation to a delayed predator-prey system with diffusion effects. With reference to the ordinary differential equations of model (1.2), Wang et al. [26] showed that if the basic reproduction ratio of system is bigger than 1 , the time delay of the immune response and the birth rate of susceptible host cells involves to a rich dynamics, which includes the occurrence of stable periodic solutions and chaotic dynamical behavior. However, there is little concern with the spatial patterns 
of delayed viral infection models with diffusion. Therefore, our main aim to explore whether time delay can drive the emergence of spatial patterns and how initial conditions affect the spatially distribution of cells density for the nonlinear system (1.2). Our analysis reveals that the delay may induce spatial patterns when the delay passes through a sequence of critical values and different initial conditions may lead to different shapes of patterns.

The organization of this paper is as follows. In Section 2 we analytically investigate the stability and instability of the endemic equilibrium $\mathbf{u}^{*}$ for system (1.2). We show that when the delay passes through the critical value, the inhomogeneous state of system (1.2) switches to the spatial patterns and the existence of local Hopf bifurcation of system (1.2) is discussed. In Section 3 , by applying the normal form theory and the center manifold reduction for functional differential equations, the conditions for determining the bifurcation direction and the stability of the bifurcating periodic solution are derived. In section 4, numerical simulations are performed to illustrate our analytical findings. A brief discussion is given in Section 5 .

\section{Delay driven spatial patterns}

In this section, we shall carry out the linear stability of the endemic equilibrium $\mathbf{u}^{*}$. Set $\mathbf{u}(\mathbf{x}, t)=\mathbf{u}^{*}+\delta \mathbf{u}^{*} \exp (s t) \exp (i \mathbf{k} \cdot \mathbf{x})$, where $s$ is the eigenvalue and $k$ is the wave number. We have the following characteristic equation

$$
\operatorname{det}\left(\begin{array}{lll}
s+d_{1} k^{2}+d+\beta u_{2}^{*} & \beta u_{1}^{*} & 0 \\
-\beta u_{2}^{*} & s+d_{2} k^{2} & p u_{2}^{*} \\
0 & -c e^{-s \tau} & s+d_{3} k^{2}+b
\end{array}\right)=0
$$

Then, the characteristic equation of system (1.2) at the endemic equilibrium $\mathbf{u}^{*}$ is of the form

$$
s^{3}+a_{1} s^{2}+a_{2} s+a_{3}+\left(b_{1} s+b_{2}\right) e^{-s \tau}=0,
$$

where

$$
\begin{aligned}
& a_{1}=b+d+\beta u_{2}^{*}+\left(d_{1}+d_{2}+d_{3}\right) k^{2}, \\
& a_{2}=d_{2} k^{2}\left(b+d_{3} k^{2}\right)+\left(d+\beta u_{2}^{*}+d_{1} k^{2}\right)\left(b+d_{2} k^{2}+d_{3} k^{2}\right)+\beta^{2} u_{1}^{*} u_{2}^{*}, \\
& a_{3}=d_{2} k^{2}\left(d+\beta u_{2}^{*}+d_{1} k^{2}\right)\left(b+d_{3} k^{2}\right)+\beta^{2} u_{1}^{*} u_{2}^{*}\left(b+d_{3} k^{2}\right), \\
& b_{1}=c p u_{2}^{*}, \quad b_{2}=\left(d+\beta u_{2}^{*}+d_{1} k^{2}\right) c p u_{2}^{*} .
\end{aligned}
$$

It is well known that the endemic equilibrium $\mathbf{u}^{*}$ is unstable if there is at least one root with $\operatorname{Re} s>0$, and is stable if Res $<0$ for all $s$. That is to say, the stability of solutions depends on the location of the zeros of the associated characteristic equation. Thus, the delay destabilizes endemic equilibrium if the critical point $\tau$ satisfies $\operatorname{Re} s=0$, which is called delay driven instability.

Clearly, when $\tau=0$, equation (2.1) becomes

$$
s^{3}+a_{1} s^{2}+\left(a_{2}+b_{1}\right) s+a_{3}+b_{2}=0
$$

since $a_{1}>0, a_{3}+b_{2}>0$ and $a_{1}\left(a_{2}+b_{1}\right)-\left(a_{3}+b_{2}\right)>0$, we have that all roots of equation (2.2) have negative real roots. Hence, by the Routh-Hurwitz 
theorem, the endemic equilibrium $\mathbf{u}^{*}$ of system (1.2) is asymptotically stable when $\tau=0$.

Now, we discuss the effects of the delay on the stability of the endemic equilibrium $\mathbf{u}^{*}$. If $s=i w(w>0)$ is a root of equation (2.1), separating real and imaginary parts, it follows that

$$
\left\{\begin{array}{l}
a_{1} w^{2}-a_{3}=b_{1} w \sin w \tau+b_{2} \cos w \tau \\
w^{3}-a_{2} w=b_{1} w \cos w \tau-b_{2} \sin w \tau
\end{array}\right.
$$

Squaring and adding the two equations of (2.3), we derive that

$$
w^{6}+\left(a_{1}^{2}-2 a_{2}\right) w^{4}+\left(a_{2}^{2}-2 a_{1} a_{3}-b_{1}^{2}\right) w^{2}+a_{3}^{2}-b_{2}^{2}=0 .
$$

Letting $z=w^{2}$ gives

$$
z^{3}+\left(a_{1}^{2}-2 a_{2}\right) z^{2}+\left(a_{2}^{2}-2 a_{1} a_{3}-b_{1}^{2}\right) z+a_{3}^{2}-b_{2}^{2}=0 .
$$

Due to the complexity of equations (2.5), we investigate their solutions numerically in the numerical section. We assume that (2.5) has at most three positive roots denoted by $z_{1}, z_{2}, z_{3}$, respectively. Then (2.4) has at most three positive roots $w_{1}=\sqrt{z_{1}}, w_{2}=\sqrt{z_{2}}, w_{3}=\sqrt{z_{3}}$. Moreover, equation (2.1) has the pure imaginary root $s=i w_{j}(j=1,2,3)$ when

$$
\tau=\tau_{j}^{(n)}=\tau_{j}^{(0)}+\frac{2 n \pi}{w_{j}}, n=0,1,2, \cdots,
$$

where $\tau_{j}^{(0)}=\frac{1}{w_{j}} \arccos \frac{b_{1}\left(w_{j}\right)^{4}+\left(a_{1} b_{2}-a_{2} b_{1}\right)\left(w_{j}\right)^{2}-a_{3} b_{2}}{b_{2}^{2}+b_{1}^{2}\left(w_{j}\right)^{2}}$.

Let us denote

$$
\tau^{*}=\tau_{j 0}^{(0)}=\min _{j=1,2,3}\left\{\tau_{j}^{(0)}\right\}, \quad w^{*}=w_{j 0} .
$$

From above arguments, we have the following results.

Theorem 1. For system (1.2), the following statements are true:

(i) If $\tau=0$, then the endemic equilibrium $\mathbf{u}^{*}$ is asymptotically stable. Hence, spatial patterns can not be generated without the delay.

(ii) If $\tau>0$, then there exists a critical point $\tau^{*}$ such that the endemic equilibrium $\mathbf{u}^{*}$ is asymptotically stable for $\tau \in\left[0, \tau^{*}\right)$ and unstable for $\tau \in\left(\tau^{*}, \infty\right)$.

Theorem 2. System (1.2) undergoes Hopf bifurcation at the endemic equilibrium $\mathbf{u}^{*}$ when $\tau=\tau^{*}$.

Proof. Substituting $s=\sigma+i w$ into (2.1), we have

$$
\left\{\begin{array}{l}
\sigma^{3}-3 \sigma w^{2}+a_{1} \sigma^{2}-a_{1} w^{2}+a_{2} \sigma+a_{3}+e^{-\sigma \tau}\left[\left(b_{1} \sigma+b_{2}\right) \cos \tau w\right. \\
\left.\quad+b_{1} w \sin \tau w\right]=0, \\
3 \sigma^{2} w-w^{3}+2 a_{1} \sigma w+a_{2} w+e^{-\sigma \tau}\left[b_{1} w \cos \tau w-\left(b_{1} \sigma+b_{2}\right) \sin \tau w\right]=0 .
\end{array}\right.
$$


Differentiating the above equations with respect to $\tau$, after substituting $\sigma=0$ into it, we obtain

$$
\left\{\begin{array}{r}
X \frac{d \sigma}{d \tau}+Y \frac{d w}{d \tau}=U \\
-Y \frac{d \sigma}{d \tau}+X \frac{d w}{d \tau}=V
\end{array}\right.
$$

where

$$
\begin{aligned}
& X=-3 w^{2}+a_{2}+\cos w \tau\left(b_{1}-b_{2} \tau\right)-b_{1} w \tau \sin w \tau \\
& Y=-2 a_{1} w+b_{1} w \tau \cos w \tau+\sin w \tau\left(b_{1}-b_{2} \tau\right) \\
& U=b_{2} w \sin w \tau-b_{1} w^{2} \cos w \tau, \quad V=b_{2} w \cos w \tau+b_{1} w^{2} \sin w \tau .
\end{aligned}
$$

Substituting $\tau=\tau^{*}, w=w^{*}$ and (2.3) into the above equation, we have $\left.\frac{d \sigma}{d \tau}\right|_{\tau=\tau^{*}, w=w^{*}}>0$. Therefore, the transversality condition is satisfied. Moreover, (2.1) has a pair of simple purely imaginary root $s= \pm i w^{*}$ at $\tau=\tau^{*}$. Thus, Hopf bifurcation occurs at $\tau=\tau^{*}$.

\section{Direction and stability of the Hopf bifurcation}

In this section, by following the idea of Hassard [9], we shall explore the direction and stability of the periodic solutions bifurcating from the positive equilibrium $\mathbf{u}^{*}$ when the delay passes through the critical value $\tau^{*}$.

Let $v_{i}=u_{i}-u^{*}$ for $i=1,2,3, \tau=\tau^{*}+\gamma, \gamma \in R$. Then system (1.2) can be written as a functional differential equation in $C=C\left([-1,0], R^{3}\right)$

$$
\dot{\mathbf{v}}(t)=L_{\gamma}\left(\mathbf{v}_{t}\right)+F\left(\gamma, \mathbf{v}_{t}\right),
$$

where $\mathbf{v}=\left(v_{1}, v_{2}, v_{3}\right)^{T}$, and $\mathbf{v}_{t}=\mathbf{v}(t+\theta), \theta \in[-1,0] . \quad L_{\gamma}: C \rightarrow R$ and $F: R \times C \rightarrow R$ are defined, respectively, by

$$
\begin{aligned}
L_{\gamma}(\phi) & =\left(\tau^{*}+\gamma\right)\left(\begin{array}{ccc}
-d_{1} k^{2}-d-\beta u_{2}^{*} & -\beta u_{1}^{*} & 0 \\
\beta u_{2}^{*} & -d_{2} k^{2} & -p u_{2}^{*} \\
0 & 0 & -d_{3} k^{2}-b
\end{array}\right)\left(\begin{array}{l}
\phi_{1}(0) \\
\phi_{2}(0) \\
\phi_{3}(0)
\end{array}\right) \\
& +\left(\tau^{*}+\gamma\right)\left(\begin{array}{lll}
0 & 0 & 0 \\
0 & 0 & 0 \\
0 & c & 0
\end{array}\right)\left(\begin{array}{c}
\phi_{1}(-1) \\
\phi_{2}(-1) \\
\phi_{3}(-1)
\end{array}\right), \\
F(\gamma, \phi) & =\left(\tau^{*}+\gamma\right)\left(\begin{array}{l}
-\beta \phi_{1}(0) \phi_{2}(0) \\
\beta \phi_{1}(0) \phi_{2}(0)-p \phi_{2}(0) \phi_{3}(0) \\
0
\end{array}\right),
\end{aligned}
$$

where $\phi(\theta)=\left(\phi_{1}(\theta), \phi_{2}(\theta), \phi_{3}(\theta)\right)^{T} \in C$. By the Riesz representation theorem, there exists a $3 \times 3$ matrix function $\rho(\theta, \gamma)$ whose components are bounded variation for $\theta \in[-1,0]$, such that

$$
L_{\gamma}(\phi)=\int_{-1}^{0} \mathrm{~d} \rho(\theta, \gamma) \phi(\theta) .
$$


In fact, we can choose

$$
\begin{aligned}
\rho(\theta, \gamma) & =\left(\tau^{*}+\gamma\right)\left(\begin{array}{lll}
-d_{1} k^{2}-d-\beta u_{2}^{*} & -\beta u_{1}^{*} & 0 \\
\beta u_{2}^{*} & -d_{2} k^{2} & -p u_{2}^{*} \\
0 & 0 & -d_{3} k^{2}-b
\end{array}\right) \delta(\theta) \\
& -\left(\tau^{*}+\gamma\right)\left(\begin{array}{lll}
0 & 0 & 0 \\
0 & 0 & 0 \\
0 & c & 0
\end{array}\right) \delta(\theta+1),
\end{aligned}
$$

where $\delta$ is delta function. For $\phi \in C=C^{1}\left([-1,0], R^{3}\right)$, define

$$
A(\gamma) \phi=\left\{\begin{array}{l}
\frac{\mathrm{d} \phi(\theta)}{\mathrm{d} \theta}, \theta \in[-1,0), \\
\int_{-1}^{0} \mathrm{~d} \rho(s, \gamma) \phi(s), \theta=0,
\end{array} \quad R(\gamma) \phi=\left\{\begin{array}{l}
0, \theta \in[-1,0), \\
F(\gamma, \phi), \theta=0
\end{array}\right.\right.
$$

Then system (3.1) can be written as

$$
\dot{\mathbf{v}}_{t}=A(\gamma) \mathbf{v}_{t}+R(\gamma) \mathbf{v}_{t} .
$$

For $\phi \in C=C^{1}\left([-1,0], R^{3}\right), \varphi \in C^{*}=C^{1}\left([0,1],\left(R^{3}\right)^{*}\right)$, we define the adjoint operator $A^{*}$ of $A(0)$ by

$$
A^{*} \varphi(s)=\left\{\begin{array}{l}
\frac{-\mathrm{d} \varphi(s)}{\mathrm{d} s}, s \in(0,1] \\
\int_{-1}^{0} \mathrm{~d} \rho^{T}(t, 0) \varphi(-t), s=0
\end{array}\right.
$$

and the adjoint bilinear form by

$$
<\varphi, \phi>=\bar{\varphi}(0) \phi(0)-\int_{-1}^{0} \int_{\xi=0}^{\theta} \bar{\varphi}(\xi-\theta) \mathrm{d} \rho(\theta, 0) \phi(\xi) \mathrm{d} \xi .
$$

In terms of the discussion in previous section, we see that $\pm i w^{*} \tau^{*}$ are eigenvalues of $A(0)$ and $A^{*}$. Next we calculate the eigenvector $q(\theta)$ of $A(0)$ corresponding to $i w^{*} \tau^{*}$ and the eigenvector $q^{*}(s)$ of $A^{*}$ belonging to $-i w^{*} \tau^{*}$, respectively. By the definition of $A(0)$ and $A^{*}$, we can obtain

$$
q(\theta)=\left(1, q_{1}, q_{2}\right)^{T} e^{i w^{*} \tau^{*} \theta}, \quad q^{*}(s)=D\left(1, q_{1}^{*}, q_{2}^{*}\right)^{T} e^{i w^{*} \tau^{*} s},
$$

where

$$
\begin{aligned}
q_{1}=-\frac{d_{1} k^{2}+d+\beta u_{2}^{*}+i w^{*}}{\beta u_{1}^{*}}, & q_{2}=\frac{-c e^{-i w^{*} \tau^{*}}\left(d_{1} k^{2}+d+\beta u_{2}^{*}+i w^{*}\right)}{\beta u_{1}^{*}\left(d_{3} k^{2}+b+i w^{*}\right)}, \\
q_{1}^{*}=\frac{d_{1} k^{2}+d+\beta u_{2}^{*}-i w^{*}}{\beta u_{2}^{*}}, & q_{2}^{*}=\frac{-p\left(d_{1} k^{2}+d+\beta u_{2}^{*}-i w^{*}\right)}{\beta\left(d_{3} k^{2}+b-i w^{*}\right)} .
\end{aligned}
$$

It follows from (3.4) that

$$
\begin{aligned}
<q^{*}, q> & =\bar{q}^{*}(0) q(0)-\int_{-1}^{0} \int_{\xi=0}^{\theta} \bar{q}^{*}(\xi-\theta) \mathrm{d} \rho(\theta, 0) q(\xi) \mathrm{d} \xi \\
& =\bar{D}\left\{1+q_{1} \bar{q}_{1}^{*}+q_{2} \bar{q}_{2}^{*}+\tau^{*}\left(1, \bar{q}_{1}^{*}, \bar{q}_{2}^{*}\right)\left(\begin{array}{ccc}
0 & 0 & 0 \\
0 & 0 & 0 \\
0 & c & 0
\end{array}\right)\left(\begin{array}{l}
1 \\
q_{1} \\
q_{2}
\end{array}\right) e^{-i w^{*} \tau^{*}}\right\} \\
& =\bar{D}\left(1+q_{1} \bar{q}_{1}^{*}+q_{2} \bar{q}_{2}^{*}+\tau^{*} c q_{1} \bar{q}_{2}^{*} e^{-i w^{*} \tau^{*}}\right)
\end{aligned}
$$


We choose $\bar{D}=\left(1+q_{1} \bar{q}_{1}^{*}+q_{2} \bar{q}_{2}^{*}+\tau^{*} c q_{1} \bar{q}_{2}^{*} e^{-i w^{*} \tau^{*}}\right)^{-1}$, such that $\left\langle q^{*}, q>=1\right.$ and $\left\langle q^{*}, \bar{q}\right\rangle=0$, where $\bar{D}$ is the conjugate imaginary.

In the following, we compute the coordinates describing center manifold $C_{0}$ at $\gamma=0$. Define

$$
z(t)=<q^{*}, \mathbf{v}_{t}>, \quad W(t, \theta)=\mathbf{v}_{t}(\theta)-z(t) q(\theta)-\overline{z(t) q(\theta)},
$$

therefore,

$$
\mathbf{v}_{t}(\theta)=W(t, \theta)+z(t)\left(1, q_{1}, q_{2}\right)^{T} e^{i w^{*} \tau^{*} \theta}+\bar{z}(t)\left(1, \bar{q}_{1}, \bar{q}_{2}\right)^{T} e^{-i w^{*} \tau^{*} \theta} .
$$

On the center manifold $C_{0}$, we have

$$
W(t, \theta)=W(z(t), \bar{z}(t), \theta)=W_{20} \frac{z^{2}}{2}+W_{11} z \bar{z}+W_{02} \frac{\bar{z}^{2}}{2}+\cdots,
$$

where $z$ and $\bar{z}$ are local coordinates for the center manifold $C_{0}$ in the direction of $q^{*}$ and $\bar{q}^{*}$. Note that $W$ is real if $\mathbf{v}_{t}$ is real. We can only consider real solutions. For the solution $\mathbf{v}_{t} \in C_{0}$ when $\gamma=0$, by (3.2)-(3.6), we have

$$
\begin{aligned}
\dot{z}(t) & =i w^{*} \tau^{*} z(t)+<q^{*}, R(0)(W(z, \bar{z}, \theta)+2 \operatorname{Re}\{z(t) q(\theta)\})> \\
& =i w^{*} \tau^{*} z(t)+\bar{q}^{*}(0) F(0, W(z, \bar{z}, 0)+2 \operatorname{Re}\{z(t) q(0)\}) \\
& =i w^{*} \tau^{*} z(t)+g(z, \bar{z}),
\end{aligned}
$$

where

$$
\begin{aligned}
g(z, \bar{z}) & =\bar{q}^{*}(0) F(0, W(z, \bar{z}, 0)+2 \operatorname{Re}\{z(t) q(0)\}) \\
& =g_{20} \frac{z^{2}}{2}+g_{11} z \bar{z}+g_{02} \frac{\bar{z}^{2}}{2}+g_{21} \frac{z^{2} \bar{z}}{2}+\cdots .
\end{aligned}
$$

From (3.7) and (3.8), we obtain

$$
\begin{aligned}
& v_{1 t}(0)=z+\bar{z}+W_{20}^{(1)}(0) \frac{z^{2}}{2}+W_{11}^{(1)}(0) z \bar{z}+W_{02}^{(1)}(0) \frac{\bar{z}^{2}}{2}+o\left(|(z, \bar{z})|^{3}\right), \\
& v_{2 t}(0)=z q_{1}+\bar{z} \bar{q}_{1}+W_{20}^{(2)}(0) \frac{z^{2}}{2}+W_{11}^{(2)}(0) z \bar{z}+W_{02}^{(2)}(0) \frac{\bar{z}^{2}}{2}+o\left(|(z, \bar{z})|^{3}\right), \\
& v_{3 t}(0)=z q_{2}+\bar{z} \bar{q}_{2}+W_{20}^{(3)}(0) \frac{z^{2}}{2}+W_{11}^{(3)}(0) z \bar{z}+W_{02}^{(3)}(0) \frac{\bar{z}^{2}}{2}+o\left(|(z, \bar{z})|^{3}\right) .
\end{aligned}
$$

By use of the definition of $F\left(\gamma, \mathbf{v}_{t}\right)$, we have

$$
\begin{aligned}
g(z, \bar{z})= & \bar{q}^{*}(0) F(0, W(z, \bar{z}, 0)+2 \operatorname{Re}\{z(t) q(0)\}) \\
= & \tau^{*} \bar{D}\left(1, \bar{q}_{1}^{*}, \bar{q}_{2}^{*}\right)\left(\begin{array}{l}
-\beta v_{1 t}(0) v_{2 t}(0) \\
\beta v_{1 t}(0) v_{2 t}(0)-p v_{2 t}(0) v_{3 t}(0) \\
0
\end{array}\right) \\
= & \tau^{*} \bar{D}\left\{z^{2}\left[-q_{1} \beta+\bar{q}_{1}^{*}\left(q_{1} \beta-q_{1} q_{2} p\right)\right]\right. \\
& +2 z \bar{z}\left[-\operatorname{Re}\left\{q_{1}\right\} \beta+\bar{q}_{1}^{*}\left(\operatorname{Re}\left\{q_{1}\right\} \beta-\operatorname{Re}\left\{q_{1} \bar{q}_{2}\right\} p\right)\right] \\
& +\bar{z}^{2}\left[-\bar{q}_{1} \beta+\bar{q}_{1}^{*}\left(\bar{q}_{1} \beta-\bar{q}_{1} \bar{q}_{2} p\right)\right] \\
& +z^{2} \bar{z}\left[\left(\bar{q}_{1}^{*} \beta-\beta\right)\left(W_{11}^{(2)}(0)+W_{11}^{(1)}(0) q_{1}+\frac{W_{20}^{(2)}(0)}{2}+\frac{W_{20}^{(1)}(0)}{2} \bar{q}_{1}\right)\right. \\
& \left.\left.-\bar{q}_{1}^{*} p\left(W_{11}^{(3)}(0) q_{1}+W_{11}^{(2)}(0) q_{2}+\frac{W_{20}^{(3)}(0)}{2} \bar{q}_{1}+\frac{W_{20}^{(2)}(0)}{2} \bar{q}_{2}\right)\right]\right\} .
\end{aligned}
$$


By comparing coefficients with (3.10), we get

$$
\begin{aligned}
g_{20}= & 2 \tau^{*} \bar{D}\left[-q_{1} \beta+\bar{q}_{1}^{*}\left(q_{1} \beta-q_{1} q_{2} p\right)\right], \\
g_{11}= & 2 \tau^{*} \bar{D}\left[-\operatorname{Re}\left\{q_{1}\right\} \beta+\bar{q}_{1}^{*}\left(\operatorname{Re}\left\{q_{1}\right\} \beta-\operatorname{Re}\left\{q_{1} \bar{q}_{2}\right\} p\right)\right], \\
g_{02}= & 2 \tau^{*} \bar{D}\left[-\bar{q}_{1} \beta+\bar{q}_{1}^{*}\left(\bar{q}_{1} \beta-\bar{q}_{1} \bar{q}_{2} p\right)\right], \\
g_{21}= & 2 \tau^{*} \bar{D}\left[\left(\bar{q}_{1}^{*} \beta-\beta\right)\left(W_{11}^{(2)}(0)+W_{11}^{(1)}(0) q_{1}+\frac{W_{20}^{(2)}(0)}{2}+\frac{W_{20}^{(1)}(0)}{2} \bar{q}_{1}\right)\right. \\
& \left.-\bar{q}_{1}^{*} p\left(W_{11}^{(3)}(0) q_{1}+W_{11}^{(2)}(0) q_{2}+\frac{W_{20}^{(3)}(0)}{2} \bar{q}_{1}+\frac{W_{20}^{(2)}(0)}{2} \bar{q}_{2}\right)\right] .
\end{aligned}
$$

The value of $g_{21}$ is determined by $W_{20}(\theta)$ and $W_{11}(\theta)$, we still need to compute them. From (3.3) and (3.6), we have

$$
\begin{aligned}
\dot{W}(t, \theta) & =\dot{\mathbf{u}}_{t}(\theta)-2 \operatorname{Re}\{\dot{z}(t) q(\theta)\} \\
& =A(0) W+R(0)(W+2 \operatorname{Re}\{z(t) q(\theta)\})-2 \operatorname{Re}\{g(z, \bar{z}) q(\theta)\} \\
& \triangleq A(0) W+H(z, \bar{z}, \theta),
\end{aligned}
$$

where

$$
H(z, \bar{z}, \theta)=R(0)(W+2 \operatorname{Re}\{z(t) q(\theta)\})-2 \operatorname{Re}\{g(z, \bar{z}) q(\theta)\} .
$$

By the definition of $R(0)$, we get

$$
\begin{aligned}
H(z, \bar{z}, \theta) & =\left\{\begin{array}{l}
-2 \operatorname{Re}\{g(z, \bar{z}) q(\theta)\}, \theta \in[-1,0) \\
-2 \operatorname{Re}\{g(z, \bar{z}) q(0)\}+F(0, z, \bar{z}), \theta=0
\end{array}\right. \\
& \triangleq H_{20}(\theta) \frac{z^{2}}{2}+H_{11}(\theta) z \bar{z}+H_{02}(\theta) \frac{\bar{z}^{2}}{2}+\cdots
\end{aligned}
$$

On the center manifold $C_{0}$ near the origin, we have

$$
\dot{W}=W_{z} \dot{z}+W_{\bar{z}} \dot{\bar{z}}=W_{20} z \dot{z}+W_{11} \bar{z} \dot{z}+W_{11} z \dot{\bar{z}}+W_{02} \bar{z} \dot{\bar{z}}+\cdots .
$$

It follows from (3.9) and (3.11) that

$$
\left(A(0)-2 i w^{*} \tau^{*} \mathrm{I}\right) W_{20}(\theta)=-H_{20}(\theta), \quad A(0) W_{11}(\theta)=-H_{11}(\theta) .
$$

By (3.10) and (3.11) we have for $\theta \in[-1,0)$,

$$
H_{20}(\theta)=-g_{20} q(\theta)-\bar{g}_{20} \bar{q}(\theta), \quad H_{11}(\theta)=-g_{11} q(\theta)-\bar{g}_{11} \bar{q}(\theta) .
$$

From (3.12) and (3.13) for $\theta \in[-1,0)$, we get

$$
\dot{W}_{20}(\theta)=2 i w^{*} \tau^{*} W_{20}(\theta)+g_{20} q(\theta)+\bar{g}_{20} \bar{q}(\theta), \quad \dot{W}_{11}(\theta)=g_{11} q(\theta)+\bar{g}_{11} \bar{q}(\theta) .
$$

A simple computation yields

$$
\begin{aligned}
& W_{20}(\theta)=\frac{i g_{20} q(0)}{\tau^{*} w^{*}} e^{i w^{*} \tau^{*} \theta}+\frac{i \bar{g}_{20} \bar{q}(0)}{3 \tau^{*} w^{*}} e^{-i w^{*} \tau^{*} \theta}+E_{1} e^{2 i w^{*} \tau^{*} \theta} \\
& W_{11}(\theta)=-\frac{i g_{11} q(0)}{\tau^{*} w^{*}} e^{i w^{*} \tau^{*} \theta}+\frac{i \bar{g}_{11} \bar{q}(0)}{\tau^{*} w^{*}} e^{-i w^{*} \tau^{*} \theta}+E_{2}
\end{aligned}
$$


where $E_{1}, E_{2} \in R^{3}$ are bounded constant vectors.

In the following, we will compute $E_{1}$ and $E_{2}$. From (3.12), we have

$$
\begin{aligned}
& \int_{-1}^{0} \mathrm{~d} \rho(\theta, 0) W_{20}(\theta)=2 i w^{*} \tau^{*} W_{20}(0)-H_{20}(0), \\
& \int_{-1}^{0} \mathrm{~d} \rho(\theta, 0) W_{11}(\theta)=-H_{11}(0) .
\end{aligned}
$$

By (3.11), we obtain

$$
\begin{aligned}
& H_{20}(0)=-g_{20} q(0)-\bar{g}_{20} \bar{q}(0)+2 \tau^{*}\left(\begin{array}{l}
-q_{1} \beta \\
q_{1} \beta-q_{1} q_{2} p \\
0
\end{array}\right), \\
& H_{11}(0)=-g_{11} q(0)-\bar{g}_{11} \bar{q}(0)+2 \tau^{*}\left(\begin{array}{l}
-\operatorname{Re}\left\{q_{1}\right\} \beta \\
\operatorname{Re}\left\{q_{1}\right\} \beta-\operatorname{Re}\left\{q_{1} \bar{q}_{2}\right\} p \\
0
\end{array}\right) .
\end{aligned}
$$

Substituting $H_{20}(0)$ defined by (3.16) and $W_{20}(\theta)$ defined by (3.14) into (3.15), and noting that

$$
\begin{aligned}
& \left(i w^{*} \tau^{*} \mathrm{I}-\int_{-1}^{0} e^{i w^{*} \tau^{*} \theta} \mathrm{d} \rho(\theta, 0)\right) q(0)=0, \\
& \left(-i w^{*} \tau^{*} \mathrm{I}-\int_{-1}^{0} e^{-i w^{*} \tau^{*} \theta} \mathrm{d} \rho(\theta, 0)\right) \bar{q}(0)=0,
\end{aligned}
$$

we derive

$$
\left(2 i w^{*} \tau^{*} \mathrm{I}-\int_{-1}^{0} e^{2 i w^{*} \tau^{*} \theta} \mathrm{d} \rho(\theta, 0)\right) E_{1}=2 \tau^{*}\left(\begin{array}{l}
-q_{1} \beta \\
q_{1} \beta-q_{1} q_{2} p \\
0
\end{array}\right) .
$$

Solving the above linear equation for $E_{1}$, we have

$$
\begin{aligned}
E_{1}= & 2\left(\begin{array}{lll}
2 i w^{*}+d_{1} k^{2}+d+\beta u_{2}^{*} & \beta u_{1}^{*} & 0 \\
-\beta u_{2}^{*} & 2 i w^{*}+d_{2} k^{2} & p u_{2}^{*} \\
0 & -c e^{-2 i w^{*} \tau^{*}} & 2 i w^{*}+d_{3} k^{2}+b
\end{array}\right)^{-1} \\
& \times\left(\begin{array}{l}
-q_{1} \beta \\
q_{1} \beta-q_{1} q_{2} p \\
0
\end{array}\right) .
\end{aligned}
$$

Similarly, from (3.14)-(3.16), we obtain

$$
E_{2}=2\left(\begin{array}{lll}
d_{1} k^{2}+d+\beta u_{2}^{*} & \beta u_{1}^{*} & 0 \\
-\beta u_{2}^{*} & d_{2} k^{2} & p u_{2}^{*} \\
0 & -c & d_{3} k^{2}+b
\end{array}\right)^{-1}\left(\begin{array}{l}
-\operatorname{Re}\left\{q_{1}\right\} \beta \\
\operatorname{Re}\left\{q_{1}\right\} \beta-\operatorname{Re}\left\{q_{1} \bar{q}_{2}\right\} p \\
0
\end{array}\right)
$$

Substituting $E_{1}$ and $E_{2}$ into (3.14), we can calculate $W_{20}(\theta)$ and $W_{11}(\theta)$. Furthermore, $g_{21}$ can be determined. Therefore, all $g_{i j}$ have been expressed in 
terms of parameters, and we can compute the following values:

$$
\begin{aligned}
& c_{1}(0)=\frac{i}{2 \tau^{*} w^{*}}\left(g_{11} g_{20}-2\left|g_{11}\right|^{2}-\frac{\left|g_{02}\right|^{2}}{3}\right)+\frac{g_{21}}{2}, \\
& \mu_{2}=-\frac{\operatorname{Re}\left\{c_{1}(0)\right\}}{\operatorname{Re}\left\{s^{\prime}\left(\tau^{*}\right)\right\}}, \quad \beta_{2}=2 \operatorname{Re}\left\{c_{1}(0)\right\}, \\
& T_{2}=-\frac{\operatorname{Im}\left\{c_{1}(0)\right\}+\mu_{2} \operatorname{Im}\left\{s^{\prime}\left(\tau^{*}\right)\right\}}{\tau^{*} w^{*}},
\end{aligned}
$$

where $\mu_{2}$ determines the directions of the Hopf bifurcation, $\beta_{2}$ determines the stability of the bifurcating periodic solutions on the center manifold, and $T_{2}$ determines the period of the bifurcating periodic solutions. Thus based on these values, we have the following conclusion.

Theorem 3. At the threshold value $\tau^{*}$, where $\tau^{*}$ is denoted by (2.6). if $\mu_{2}>$ $0(<0)$, the direction of the Hopf bifurcation is forward (backward), that is, the bifurcating periodic solutions exist for $\tau>\tau^{*}\left(\tau<\tau^{*}\right)$; if $\beta_{2}<0(>0)$, the bifurcating periodic solutions are orbitally asymptotically stable (unstable); and the period increases (decreases), if $T_{2}>0(<0)$.

\section{Numerical results}

In this section, we give some numerical results based on the formulae in Section 2 and Section 3. The domain of (1.2) is confined to a square domain $\Omega=$ $[0, L] \times[0, L] \subset \mathbb{R}^{2}$ with $L=100$. The wave number for this two-dimensional domain is thereby $\mathbf{k}=2 \pi(m / L, n / L),|\mathbf{k}|=k=2 \pi \sqrt{(m / L)^{2}+(n / L)^{2}}, m, n=$ $0,1, \cdots$.

In order to the numerical simulations, we take the following values: $\lambda=$ $1000, d=0.1, \beta=0.002, a=5, p=0.05, c=0.2, b=0.3, d_{1}=d_{2}=d_{3}=$ 0.1 . For this particular choice, the positive uniform equilibrium is given by $\left(u_{1}^{*}, u_{2}^{*}, u_{3}^{*}\right)=(3838,80.2776,53.5184)$.

According to (2.5)-(2.6), we obtain that (2.5) has the only positive root $z_{1}=1.9425$ and $w=1.3937$, thus, the bifurcation threshold $\tau^{*}=0.3605$. By use of Theorem 2, the endemic equilibrium $\mathbf{u}^{*}$ is asymptotically stable when $\tau<\tau^{*}$, and unstable when $\tau>\tau^{*}$.

To check the stability and direction of the Hopf bifurcations, we compute the first Lyapunov number $c_{1}(0)$ according to Theorem 3. For $\tau^{*}=0.3605$, direct calculation gives that $c_{1}(0)=-861.92-19.01 i$, that is, $\beta_{2}>0$, which means that the bifurcation periodic solution from the spatially uniform equilibrium is stable, leading to spatially homogeneous distributions.

It is well-known that for a purely spatial homogeneous initial distribution, the system always sustains homogeneous and the spatial pattern does not generate. So we take the initial conditions with an inhomogeneous spatial perturbation. Our simulations indicate that the spiral pattern emerges. Moreover, the number of the spiral pattern depends on the number of the defect-point of the initial data. We recall that the defect-point $\left(x_{c}, y_{c}\right)$ of the initial data means that $\left(x_{c}, y_{c}\right)$ satisfied that $\left.u_{1}\left(x_{c}, y_{c}\right)\right|_{t=0}=u_{1}^{*},\left.u_{2}\left(x_{c}, y_{c}\right)\right|_{t=0}=u_{2}^{*}$ and $\left.u_{3}\left(x_{c}, y_{c}\right)\right|_{t=0}=u_{3}^{*}$. 
Here we present the results of two computer experiments differing in the form of the initial conditions.

In the first case, the initial distribution of species is given in the following form:

$$
\begin{aligned}
& u_{1}(x, y, t)=u_{1}^{*}-\epsilon_{1}(x-0.1 y-50), \\
& u_{2}(x, y, t)=u_{2}^{*}-\epsilon_{2}(y-50), \quad u_{3}(x, y, t)=u_{3}^{*},
\end{aligned}
$$

where $\epsilon_{1}=2 \times 10^{-6}, \epsilon_{2}=3 \times 10^{-6}$. In this case, the initial data contains only one defect-point $\left(x_{c}, y_{c}\right)=(55,50)$. Snapshots of $u_{1}$ are shown in Figure 1 (spatial patterns of $u_{2}$ and $u_{3}$ are qualitatively similar except for the early stages of the process when the influence of the initial condition dominates). One spiral emerges around the defect-point (Figure 1(A)). The spiral grows gradually in (Figure 1(B),(C)). Eventually, the regular spatial spiral prevails over the whole domain (Figure $1(\mathrm{D})$ ).
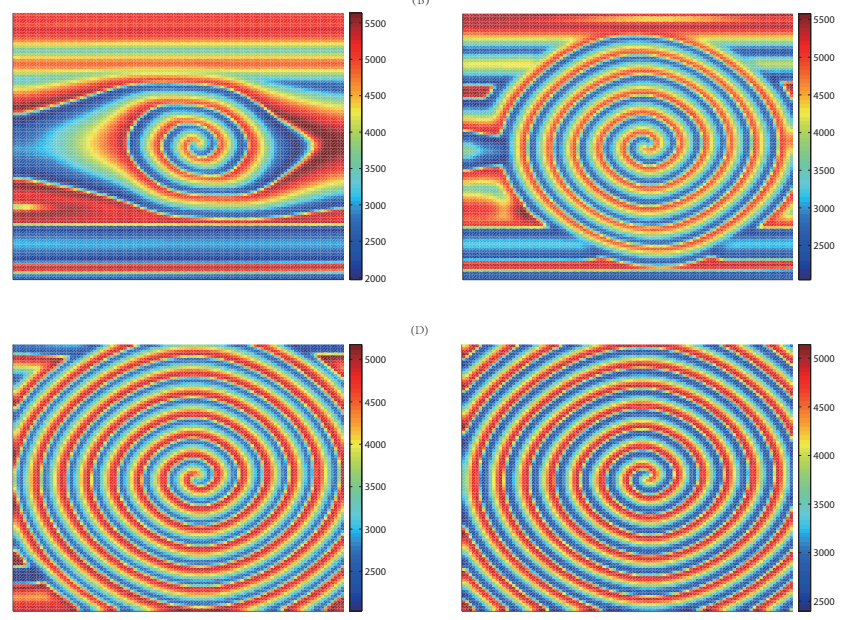

Figure 1. Example spatial patterns (snapshots of one spiral wave at different time for (A) $\mathrm{t}=400,(\mathrm{~B}) \mathrm{t}=600,(\mathrm{C}) \mathrm{t}=800,(\mathrm{D}) \mathrm{t}=1000$ ).

In the second case, the initial distribution of species is given in the following form:

$$
\begin{aligned}
& u_{1}(x, y, t)=u_{1}^{*}-\epsilon_{1}(x-20)(x-80)-\epsilon_{2}(y-30)(y-70), \\
& u_{2}(x, y, t)=u_{2}^{*}-\epsilon_{3}(y-45)-\epsilon_{4}(y-50), \quad u_{3}(x, y, t)=u_{3}^{*},
\end{aligned}
$$

where $\epsilon_{1}=2 \times 10^{-5}, \epsilon_{2}=6 \times 10^{-5}, \epsilon_{3}=3 \times 10^{-3}, \epsilon_{4}=6 \times 10^{-3}$. In this case, the initial data contains two defect-points $\left(x_{c}, y_{c}\right)=(95.735,48.333)$ and $\left(x_{c}, y_{c}\right)=(4.262,48.333)$.

Spatial patterns of $u_{1}$ are shown in Figure 2. Two spiral emerges around the defect-point (Figure 2(A)). The spiral grows gradually in (Figure 2(B),(C)). 

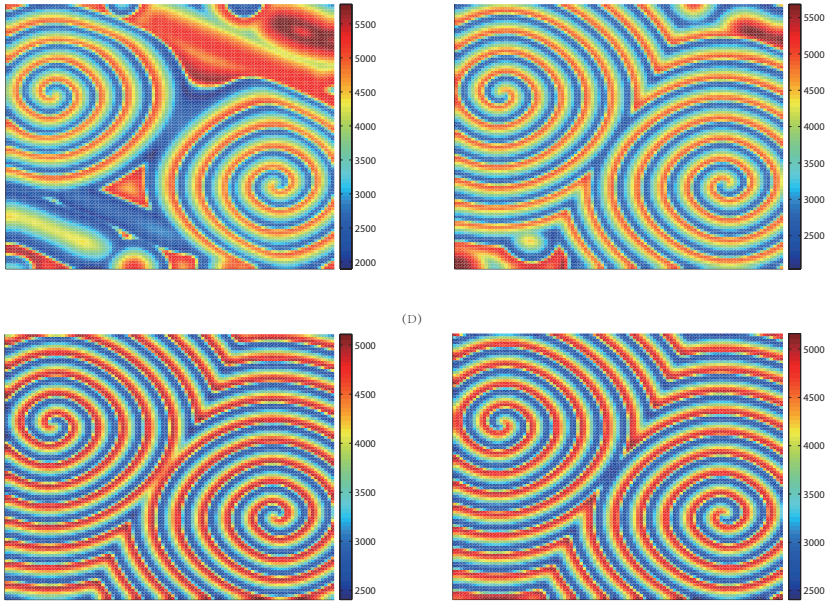

Figure 2. Example spatial patterns (snapshots of two spiral wave at different time for (A) $\mathrm{t}=400,(\mathrm{~B}) \mathrm{t}=600,(\mathrm{C}) \mathrm{t}=800,(\mathrm{D}) \mathrm{t}=1000$ ).

Eventually, the regular spatial spiral prevails over the whole domain (Figure $2(\mathrm{D}))$.

The Hopf bifurcation theorem (Theorem 3) claims that the bifurcating periodic solutions are orbitally asymptotically stable if $\operatorname{Re}\left\{c_{1}(0)\right\}<0$. Figure 3 illustrates the dynamical behavior of the spatio-temporal model in local place. We find that the emerging oscillations are periodic with respect to time.
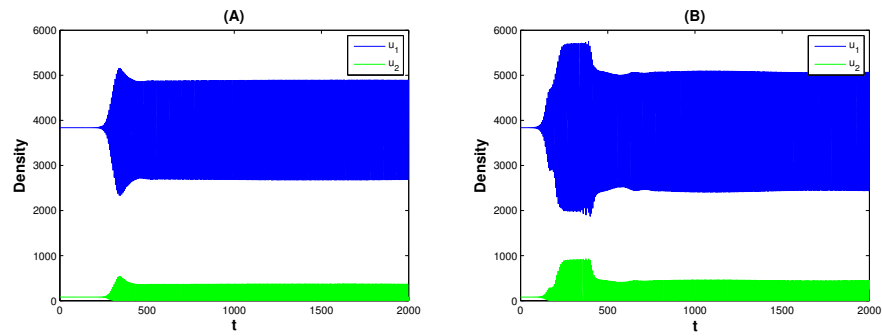

Figure 3. Dynamical behavior of the spatio-temporal model in local place. The left figure corresponds to the one spiral wave pattern. The right figure corresponds to the two spiral wave pattern.

\section{Discussions}

In this paper, we utilize a reaction diffusion equations to model a viral infection model with delayed immune response. By the bifurcation theory, we have shown that the endemic equilibrium $\mathbf{u}^{*}$ is locally asymptotically stable when the delay 
is absent, while in the case of appearance of delay the spatial pattern occurs. Here the time delay is used as a bifurcation parameter. Through analyzing the characteristic equation of the endemic equilibrium $\mathbf{u}^{*}$, we have obtained that the stability switches and Hopf bifurcation occurs when the delay passes through the critical value. In non-spatial model (1.1) Wang et al. [26] proposed that the combined effect of the strength of the lytic component, the time delay of the immune response and the birth rate of susceptible host cells is to create a rich dynamics, which includes the occurrence of stable periodic solutions and chaotic dynamical behavior and without considering the influence of initial conditions. However, we study that how the emerging spatial patterns are sensitive to initial data in spatial model (1.2). Here we present the results of two computer experiments differing in the form of the initial conditions (4.1) and (4.2). Note that the appearance of the spirals is not induced by the initial conditions. The center of each spiral is situated in a critical point $\left(x_{c}, y_{c}\right)$ satisfied that $\left.u_{1}\left(x_{c}, y_{c}\right)\right|_{t=0}=u_{1}^{*},\left.u_{2}\left(x_{c}, y_{c}\right)\right|_{t=0}=u_{2}^{*}$ and $\left.u_{3}\left(x_{c}, y_{c}\right)\right|_{t=0}=u_{3}^{*}$. The distributions (4.1) and (4.2) contain one and two such points; for other initial conditions, the number of spirals may be different. The formation of a spiral structure in the spatial distribution of virus-immune dynamics may shed new light on some old problems.

\section{Acknowledgment}

Canrong Tian acknowledges financial support by PRC grant NSFC 11201406 and by the Qinglan Project. Qunying Zhang acknowledges financial support by PRC grant NSFC 11501494.

\section{References}

[1] B. Andreianov, M. Bendahmane and R. Ruiz-Baier. Analysis of a finite volume method for a cross-diffusion model in population dynamics. Mathematical Models and Methods in Applied Sciences, 21(02):307-344, 2011. http://dx.doi.org/10.1142/S0218202511005064.

[2] Ch. Bartholdy, J.P. Christensen, D.Wodarz and A.R. Thomsen. Persistent virus infection despite chronic cytotoxic T-lymphocyte activation in Gamma interferon-deficient mice infected with lymphocytic choriomeningitis virus. Journal of Virology, 74(22):10304-10311, 2000. http://dx.doi.org/10.1128/JVI.74.22.10304-10311.2000.

[3] M. Bendahmane, T. Lepoutre, A. Marrocco and B. Perthame. Conservative cross diffusions and pattern formation through relaxation. J. Math. Pures Appl., 92(6):651-667, 2009. http://dx.doi.org/10.1016/j.matpur.2009.05.003.

[4] S. Bonhoeffer, J.M. Coffin and M.A. Nowak. Human immunodeficiency virus drug therapy and virus load. J. Virol., 71:3275-3278, 1997.

[5] S. Bonhoeffer, R.M. May, G.M. Shaw and M.A. Nowak. Virus dynamics and drug therapy. Proceedings of the National Academy of Sciences of the United States of America, 94(13):6971-6976, 1997.

[6] T. Faria. Stability and bifurcation for a delayed predator-prey model and the effect of diffusion. Journal of Mathematical Analysis and Applications, 254(2):433463, 2001. http://dx.doi.org/10.1006/jmaa.2000.7182. 
[7] G.A. Funka, V.A.A. Jansen, S. Bonhoeffer and T. Killingback. Spatial models of virus-immune dynamics. Journal of Theoretical Biology, 233(2):221-236, 2005. http://dx.doi.org/10.1016/j.jtbi.2004.10.004.

[8] H. Gómez-Acevedo, M.Y. Li and S. Jacobson. Multistability in a model for CTL response to HTLV-I infection and its implications to HAM/TSP development and prevention. Bulletin of Mathematical Biology, 72(3):681-696, 2010. http://dx.doi.org/10.1007/s11538-009-9465-z.

[9] B.D. Hassard, N.D. Kazarinoff and Y.H. Wan. Theory and Applications of Hopf Bifurcation. Cambridge University Press, Cambridge, 1981.

[10] S. Iwami, S. Nakaoka and Y. Takeuchi. Viral diversity limits immune diversity in asymptomatic phase of HIV infection. Theoretical Population Biology, 73(3):332-341, 2008. http://dx.doi.org/10.1016/j.tpb.2008.01.003.

[11] O. Krakovska and L.M. Wahl. Optimal drug treatment regimens for HIV depend on adherence. Journal of Theoretical Biology, 246(3):499-509, 2007. http://dx.doi.org/10.1016/j.jtbi.2006.12.038.

[12] M.N. Kuperman and H.S. Wio. Front propagation in epidemiological models with spatial dependence. Physica A: Statistical Mechanics and its Applications, 272(1-2):206-222, 1999. http://dx.doi.org/10.1016/S0378-4371(99)00284-8.

[13] H.-D. Kwon. Optimal treatment strategies derived from a HIV model with drug-resistant mutants. Appl. Math. Comp., 188(2):1193-1204, 2007. http://dx.doi.org/10.1016/j.amc.2006.10.071.

[14] M.Y. Li and H. Shu. Global dynamics of a mathematical model for HTLV-I infection of $\mathrm{CD}^{+} \mathrm{T}$ cells with delayed CTL response. Nonlinear Analysis: Real World Applications, 13(3):1080-1092, 2012. http://dx.doi.org/10.1016/j.nonrwa.2011.02.026.

[15] J. Liu and Z. Lin. Stationary patterns for a predator-prey model with Holling type III response function and cross-diffusion. Bulletin of the Korean mathematical Society, 47(2):251-261, 2010. http://dx.doi.org/10.4134/BKMS.2010.47.2.251.

[16] A. Matzavinos and M.A.J. Chaplain. Travelling-wave analysis of a model of the immune response to cancer. Comptes Rendus Biologies, 327(11):995-1008, 2004. http://dx.doi.org/10.1016/j.crvi.2004.07.016.

[17] M.A. Nowak, S. Bonhoeffer, A.M. Hill, R. Boehme, H.C. Thomas and H. McDade. Viral dynamics in hepatitis B virus infection. Proceedings of the National Academy of Sciences, 93(9):4398-4402, 1996.

[18] R. Peng and M. Wang. Pattern formation in the Brusselator system. Journal of Mathematical Analysis and Applications, 309(1):151-166, 2005. http://dx.doi.org/10.1016/j.jmaa.2004.12.026.

[19] A.S. Perelson, A.U. Neumann, M. Markowitz, J.M. Leonard and D. Ho. Hiv-1 dynamics in vivo: virion clearance rate, infected cell lifespan, and viral generation time. Science, 271(5255):1582-1586, 1996. http://dx.doi.org/10.1126/science.271.5255.1582.

[20] X. Shi, X. Zhou and X. Song. Dynamical behavior of a delay virus dynamics model with CTL immune response. Nonlinear Anal. RWA, 11(3):1795-1809, 2010. http://dx.doi.org/10.1016/j.nonrwa.2009.04.005.

[21] X. Song and S. Cheng. A delay-differential equation model of HIV infection of cd4 $4^{+}$T-cells. The Korean Mathematical Society, 42(5):1071-1086, 2005. http://dx.doi.org/10.4134/JKMS.2005.42.5.1071. 
[22] M.O. Souza and J.P. Zubelli. Global stability for a class of virus models with cytotoxic T lymphocyte immune response and antigenic variation. Bull. Math. Biol., 73(3):609-625, 2011. http://dx.doi.org/10.1007/s11538-010-9543-2.

[23] C.R. Tian. Delay-driven spatial patterns in a plankton allelopathic system. Chaos, 22(1), 2012. http://dx.doi.org/10.1063/1.3692963.

[24] C.R. Tian, L. Zhang and Z. Lin. Pattern formation for a model of plankton allelopathy with cross-diffusion. Journal of the Franklin Institute, 348(8):19471964, 2011. http://dx.doi.org/10.1016/j.jfranklin.2011.05.013.

[25] A. Turing. The chemical basis of morphogenesis. Philos. Trans. Royal Soc. B, 237(641):37-72, 1952. http://dx.doi.org/10.1098/rstb.1952.0012.

[26] K. Wang, W.D. Wang, H. Pang and X. Liu. Complex dynamic behavior in a viral model with delayed immune response. Physica D: Nonlinear Phenomena, 226(2):197-208, 2007. http://dx.doi.org/10.1016/j.physd.2006.12.001.

[27] M. Wang. Stationary patterns for a prey-predator model with prey-dependent and ratio-dependent functional responses and diffusion. Physica D: Nonlinear Phenomena, 196(1-2):172-192, 2004. http://dx.doi.org/10.1016/j.physd.2004.05.007.

[28] X. Wang and Y. Tao. Lyapunov function and global properties of virus dynamics with immune response. International Journal of Biomathematics, 01(04):443448, 2008. http://dx.doi.org/10.1142/S1793524508000382.

[29] X. Wang, Y. Tao and X. Song. Global stability of a virus dynamics model with Beddington-DeAngelis incidence and CTL immune response. Nonlinear Dynamics, 66(4):825-830, 2011. http://dx.doi.org/10.1007/s11071-011-9954-0.

[30] D. Wodarz, J.P. Christensen and A.R. Thomsen. The importance of lytic and nonlytic immune responses in viral infections. Trends in Immunology, 23(4):194200, 2002. http://dx.doi.org/10.1016/S1471-4906(02)02189-0.

[31] Q. Xie, D. Huang, S. Zhang and J. Cao. Analysis of a viral infection model with delayed immune response. Applied Mathematical Modelling, 34(9):2388-2395, 2010. http://dx.doi.org/10.1016/j.apm.2009.11.005.

[32] X.-P. Yan. Stability and Hopf bifurcation for a delayed prey-predator system with diffusion effects. Applied Mathematics and Computation, 192:552-566, 2007. http://dx.doi.org/10.1016/j.amc.2007.03.033.

[33] K. Yoshida. The hopf bifurcation and its stability for semilinear diffusion equations with time delay arising in ecology. Hiroshima Math. J., 12:321-348, 1982.

[34] Z. Yuan and X. Zou. Global threshold dynamics in an HIV virus model with nonlinear infection rate and distributed invasion and production delays. Math. Biosci. Eng., 10(2):483-498, 2013. http://dx.doi.org/10.3934/mbe.2013.10.483.

[35] J.-F. Zhang, W.-T. Li and X.-P. Yan. Multiple bifurcations in a delayed predatorprey diffusion system with a functional response. Nonlinear Analysis: RWA, 11(4):2708-2725, 2010. http://dx.doi.org/10.1016/j.nonrwa.2009.09.019. 